\title{
Traffic classification and service in all-optical networks
}

\author{
Yunhao Li, Mark Joseph Francisco, Ioannis Lambadaris, Changcheng Huang \\ \{liyunhao, mfranc, ioannis, huang\}@sce.carleton.ca \\ Advanced Optical Networking Laboratory, SCE Department, Carleton University \\ 1125 Colony By Drive, Ottawa, Ontario, Canada
}

\begin{abstract}
All-Optical Networks require end-to-end lightpaths to be established for traffic to flow, given that there are no wavelength converters present in the network. It is shown that as the lightpaths traverse more hops, the blocking probability increases. This is causes the Fairness Problem. We introduce the Traffic Classification and Service Method (ClaServ), which optimizes the Fairness Problem, as well as reduce the traffic blocking probability when the networks require lower blocking probability. The combination of the Waveband Access Range (WAR) and the Waveband Reservation (WRsv) methods changes the traffic's distribution among the wavebands on each link of the path to control the degree of the interference among the classified traffic. Under certain traffic load, by setting the range of accessible wavebands and reserved wavebands for classified traffic, the network can achieve both the fairness and low blocking probability for all type of traffic. The simulation results show that for a 4x4 Mesh-Torus network the ClaServ method can greatly reduce the blocking probability for longer lightpaths by a factor of 100 . It is also described how the ClaServ method can easily be implemented into a distributed signaling protocol.
\end{abstract}

Keywords: WDM, fairness, traffic classification, ClaServ, optical networks, blocking probability

\section{INTRODUCTION}

It has been a general trend of Internet traffic to grow at an exponential rate ${ }^{[1]}$, and thus high capacity backbone networks are migrating to wavelength routed all-optical networks ${ }^{[2]}$. In the absence of wavelength converters, the same wavelength must be available on each link of the path for establishing a lightpath; this is known as the wavelengthcontinuity constraint ${ }^{[3]}$. In the following part of this article, we assume the networks for study satisfy this constraint.

All-optical Networks require a single wavelength to be established from the source to the destination, given no wavelength converters are present. Calls for establishing a lightpath are made at the source node. A collection of calls at the source nodes is called traffic. Under the dynamic traffic pattern, the traffic for establishing a connection (lightpath) between the source node and the destination node (s-d pair) can randomly arrive to the network, and require a random length of service time. The lightpaths' length (number of hops) may vary with the network topology. The traffic for establishing long lightpath suffer higher blocking probability than the traffic for establishing short lightpath because the number of interfering sessions on a path tends to increase with the number of hops ${ }^{[4]}$. Figure 1 shows our simulation results proving this observation. In a $4 \times 4$ Mesh-Torus network with each link accommodating 240 wavelengths, as the traffic load (Erlang) approaches 400 calls per unit time, the traffic for establishing the 4-hop lightpath suffer more than $95 \%$ blocking probability while the traffic for establishing the 1-hop lightpath experiences zero blocking probability.

This phenomenon can be described as the Fairness Problem can be defined as the inability of call requests of shorter lightpaths to have an equal blocking probability as call requests of longer lightpaths. The low blocking probability of shorter lightpaths contributes to the higher blocking probability of the longer lightpaths. The solution to this problem would be to equalize the blocking probability of call requests for both short and long lightpaths.

Two methods were proposed to solve the fairness problem ${ }^{[5]}$. In the Protecting Threshold Method (Thr), the single-hop traffic is assigned an idle wavelength only if the number of idle wavelengths on the link is at or above a given threshold. The Wavelength Reservation Method (Rsv) makes a given wavelength on a specified link is dedicated to a traffic arrival stream. As showed in ${ }^{[5]}$, the Thr method deteriorates the blocking probability of the calls for establishing single-hop lightpath as well as the average call blocking probability, but only slightly reduce the blocking probability of the calls for long lightpath. The reason is that not only the amount but also the distribution of the single-hop lightpath on 
each link of the path causes the interference to the long lightpath. Not only the single-hop lightpath, but also other short lightpath can interfere with the long lightpath too.

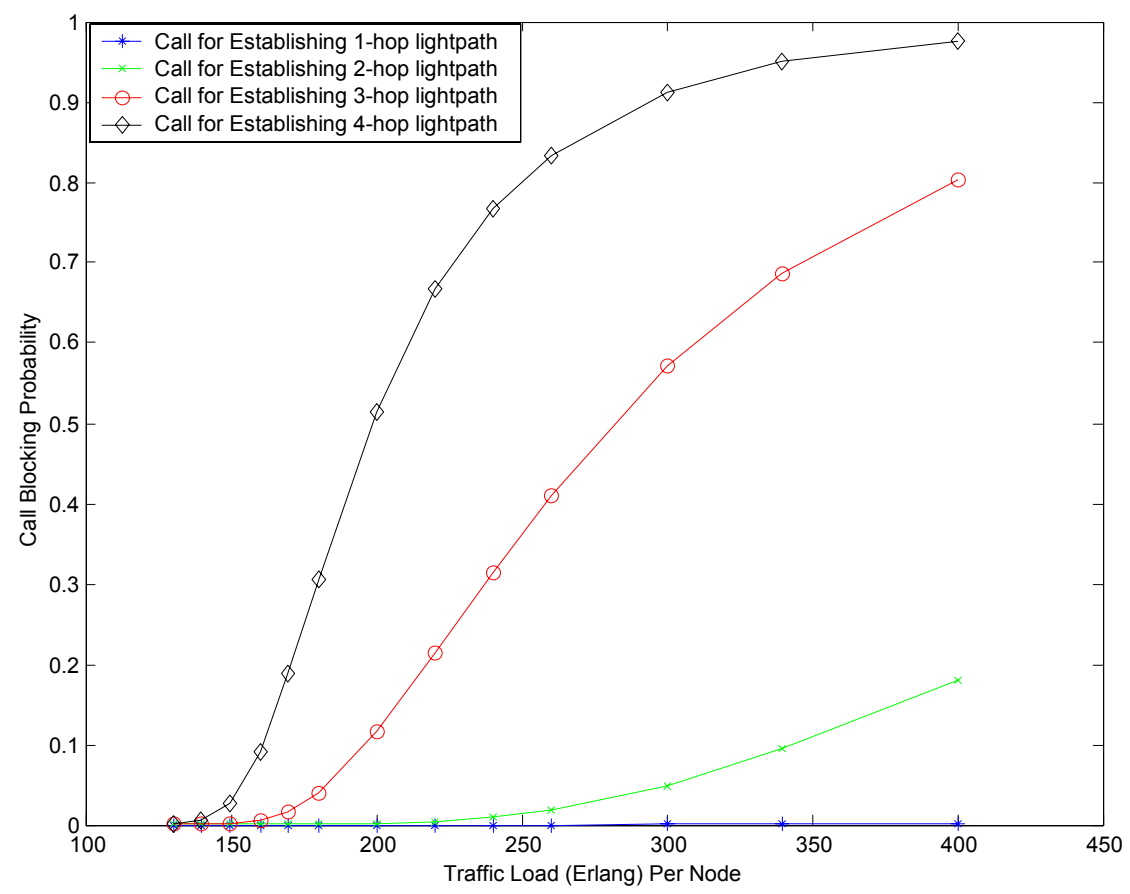

Figure 1. The blocking probability of the establishment of the lightpaths

In this paper we propose the Traffic Classification and Service Method (ClaServ) to optimize the Fairness Problem. Traffic is classified depending on the number of hops along the path from the source to the destination. The classified traffic is then offered different services to guarantee a low blocking probability for all types of traffic to optimize the fairness problem. To allow this, all the wavelengths on each link are separated equally into a set of wavebands, which are sorted indexed. Separating the wavelengths into wavebands can benefit the hierarchal searching of the available wavelengths to fast the processing for the establishment of lightpath in the Dense Wavelength Division Multiplexing (DWDM) ${ }^{[3]}$ Network. The combination of the Waveband Access Range Method (WAR) and the Waveband Reservation Method (WRsv) is used to control the interference among the classified traffic. In this paper it is assumed that wavelength routing has already been completed.

The remaining part of this article is arranged as follows. Section 2 gives the introduction of the traffic classification. Section 3 introduces the WAR and the WRsv methods as well as their service to the classified traffic. The simulation results and performance evaluation are given in Section 4. Finally, conclusions are drawn in Section 5.

\section{TRAFFIC CLASSIFICATION}

Traffic can be considered the sequence of lightpath establishment requests generated from a node in the alloptical network. The traffic are classified by the number of hops they going to traverse or traversed. This count of hops is called the traffic type and is used as the classifier of the traffic. The traffic can be classified either at the source node or at the destination node. In the first method, as traffic arrives, the source node checks the routing table to find the path from the source to the destination and count the number of hops on the path. This count of hops determines the type of traffic. The first case is called the Source Traffic Classification. For the second method, when traffic arrives, the source node grants a counter to this traffic and passes this traffic toward the destination node along the path. Each intermediate node will modify the counter when they receive the request. The value of the counter at the destination node is the type of the traffic. This case is called the Destination Traffic Classification. Destination Traffic Classification can be 
implemented into a distributed signaling protocol ${ }^{[6]}$. As shown in figure 2 (a), as the traffic arrives to one node of the network, a path is found for the source-destination pair. The source node generates a PROB packet and sends it toward the destination node along the path to collect wavelength availability information on the path. Figure 2 (b) shows the PROB packet containing a counter field whose value is initialized to zero at the source node. As the PROB packet is passed to the destination, each node along the path increments the counter field, as illustrated in figure 2 (c). At the destination, assuming the counter equals to $\mathrm{N}$, the request type is $\mathrm{N}$-hop traffic.

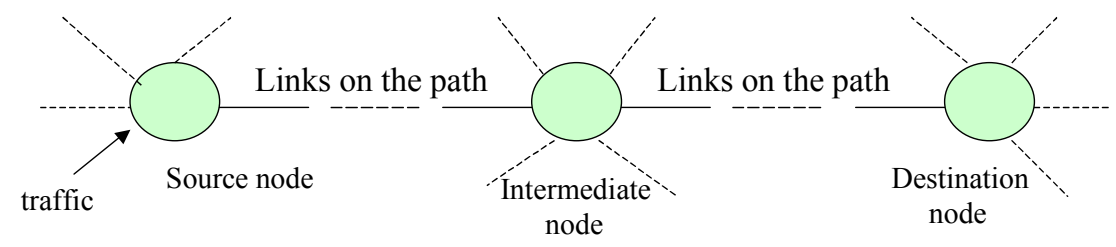

(a). Find the path for the source-destination pair when traffic arrives

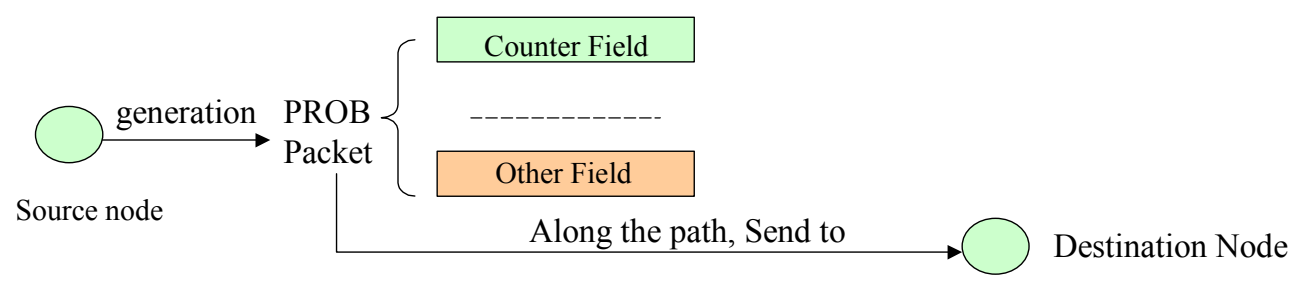

(b). The Counter Field contained by the PROB packet

\begin{tabular}{|c|c|c|c|c|}
\hline \multirow{2}{*}{ Value of the } & Source node & Intermediate node & Destination node & Traffic Type \\
\hline & 0 & Previous value +1 & Previous value $+1=\mathrm{N}$ & $\mathrm{N}$ hop Traffic \\
\hline
\end{tabular}

(c). The process of destination traffic classification

Figure 2. The implementation of the Traffic classification into the signaling protocol

\section{WAVEBAND ACCESS RANGE AND WAVEBAND RESERVATION METHODS}

The purpose of the WAR and WRsv methods is to reduce the interference of the short lightpaths to the long lightpaths when establishing lightpaths as well as guarantee the low blocking probability for all type of traffic under certain traffic load. In the $4 \times 4$ mesh-torus network, short lightpaths can be considered as 1-hop or 2-hops in length, whereas anything larger is a long lightpath. The following two subsections describe the WAR method and the WRsv method in detail.

\subsection{Waveband access range method}

The Waveband Access Range Method (WAR) is a method of assigning wavelength to lightpath requests. This involves the division of wavelengths into wavebands and assigning a waveband access range to a type of traffic. The waveband access range for different type of traffic can be different. For certain traffic, it can only assign the available wavelength to the path from its accessible wavebands. Normally, the traffic with small number of hops has less 
waveband access range than the traffic with large number of hops. By changing the way of the traffic's distribution on each link of the path, the WAR method reduces the interference for the traffic with large number of hops from the traffic with small number of hops. An example of WAR is showed in figure 3, assuming there are $\mathrm{K}$ wavebands on each link, the 1-hop traffic only can access the first $\mathrm{X}$ wavebands. The 2-hop traffic can access the first $\mathrm{Y}$ wavebands. All the other type traffic can access all the $\mathrm{K}$ wavebands.

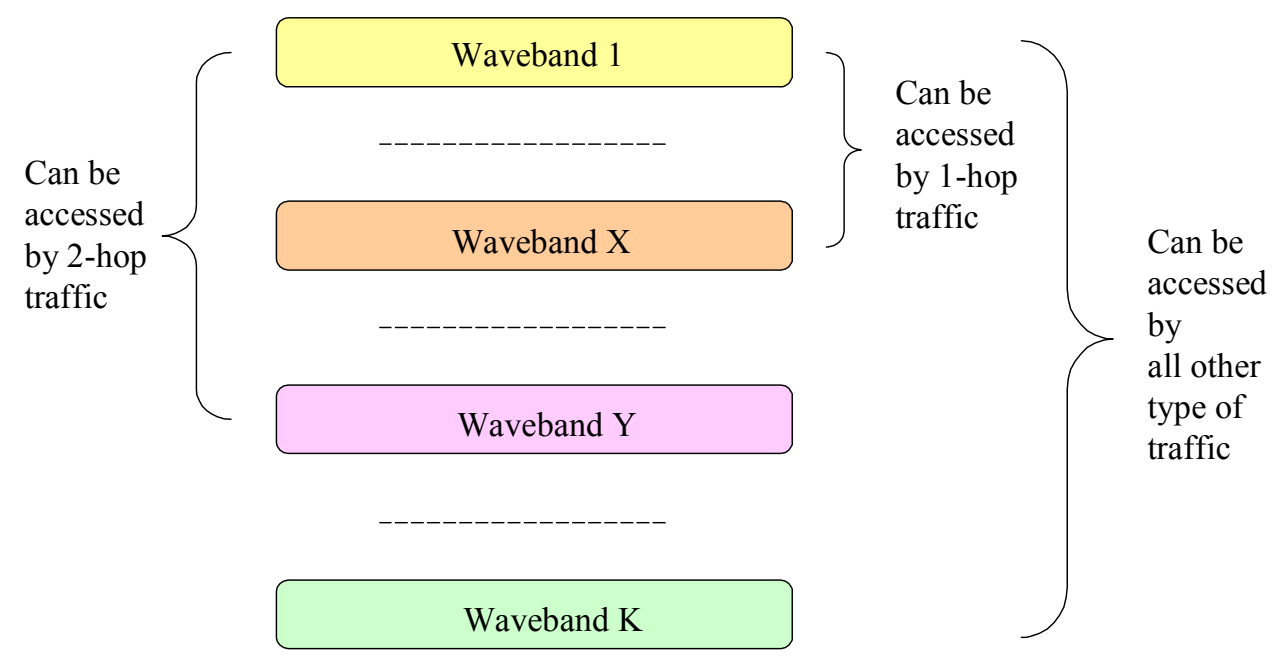

Figure 3. Waveband Access Range Method

\subsection{Waveband reservation method}

Under certain traffic load, even though the traffic with small number of hops (the 1-hop or 2-hop traffic in a 16 node mesh-torus network) experiences small blocking probability, their interference can cause the high blocking probability of the traffic with large number of hops (the 3-hop or 4-hop traffic in a 16 node mesh-torus network). The Waveband Reservation Method (WRsv) is used to puts up barriers to sharing the wavelength resource. WRsv reserves one or more wavebands for the traffic with large number of hops. If the traffic could not find the available wavelength from the unreserved wavebands, it will search the reserved wavebands for available wavelengths. Under certain traffic load, by setting the number of wavebands reserved for certain type of traffic stream, the interference is reduced for the traffic with large number of hops while the traffic with small number of hops still experience small blocking probability. Figure 4 shows one example of the division of the wavebands. The waveband $\mathrm{k}$ is reserved for 4-hop traffic; the waveband $\mathrm{Y}+1$ to $\mathrm{K}-1$ are reserved for 3-hop and 4-hop traffic. The first $\mathrm{Y}$ wavebands are unreserved. A request for longer lightpaths is forced to search the unreserved wavebands before searching the reserved wavebands. This causes some competition for the unreserved wavebands, but guarantees wavebands available for the longer lightpaths, given there are wavelengths. 


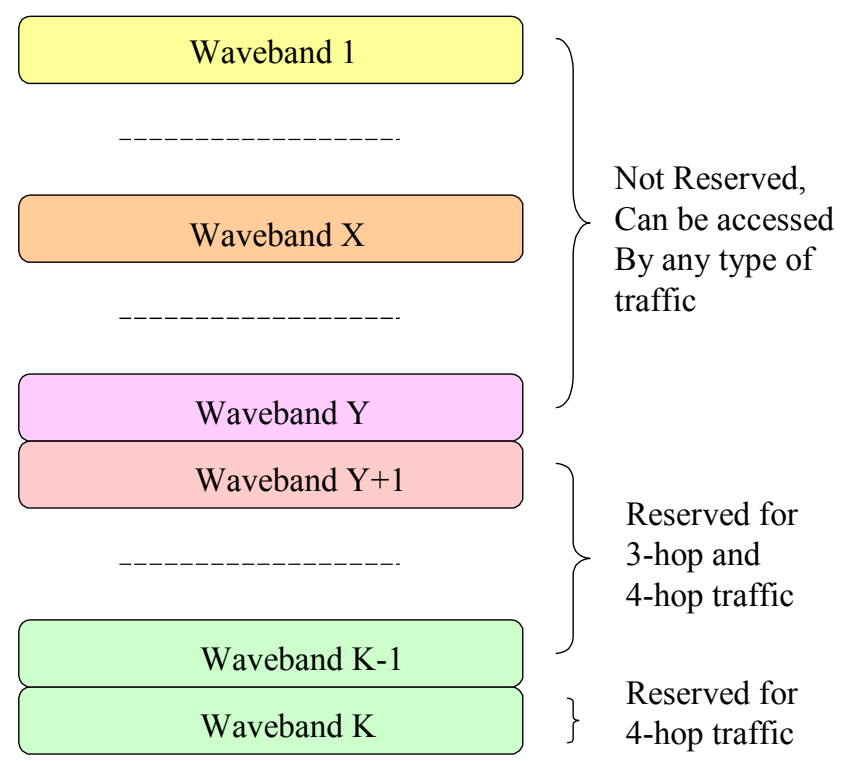

Figure 4. Waveband Reservation

\subsection{The combination of the WAR and WRsv methods}

Both of the WAR and WRsv methods can be used the same time to achieve the low blocking probability for all type of traffic. For the traffic with small number of hops, the WAR method limits their interference to other type of traffic. For the traffic with large number of hops, the WRsv method guarantees the low interference from other type of traffic. The combination of the WAR and WRsv methods serves the classified traffic to guarantee the degree of the interference one type of traffic received from other type of traffic or the degree of interference one type of traffic contributes to other type of traffic. In this way, the interference among the traffic is under control, the fairness can be achieved for all type of traffic.

\section{SIMULATION RESULTS AND PERFORMANCE EVALUATION}

Simulation experiments are used to evaluate the ClaServ Method and compared the networks' performance with and without employ the ClaServ Method. We used OPNET Modeler as our simulation tool. We compared the performance of the networks in two aspects (i) the fairness achievement, and (ii) the network average call blocking probability. We studied the $4 \times 4$ Mesh-Torus Network, depicted in figure 5. This network topology can have 1-hop, 2hop, 3-hop, and 4-hop type of traffic. The following parameters were used in the simulations. (i) Each link has 240 bidirectional wavelengths available when no lightpaths exist; (ii) The destinations of the lightpaths are equally distributed; (iii) The requests are random and follow a Poisson Distribution; (iv) The call holding time (lightpath duration) is exponentially distributed with a mean of one time unit; (v) The shortest path routing algorithms is used to allocate the path for the s-d pair; (vi) It is assumed that there is no delay between nodes; (vii) There are no wavelength converters in the network, so the Wavelength Continuity Constraint is valid; (viii) The Random wavelength assignment algorithm is used for assigning the wavelength to the path ${ }^{[7]}$.

As shown in Figure 6, the 240 wavelengths on each link are separated into 8 wavebands. Each waveband contains 30 wavelengths. For performing the ClaServ method, waveband 1,2,3, and 4 can be accessed by all types of 
traffic, waveband 5 and 6 can be accessed by 2-hop, 3-hop and 4-hop traffic, waveband 7 and 8 are reserved for 3-hop and 4-hop traffic.

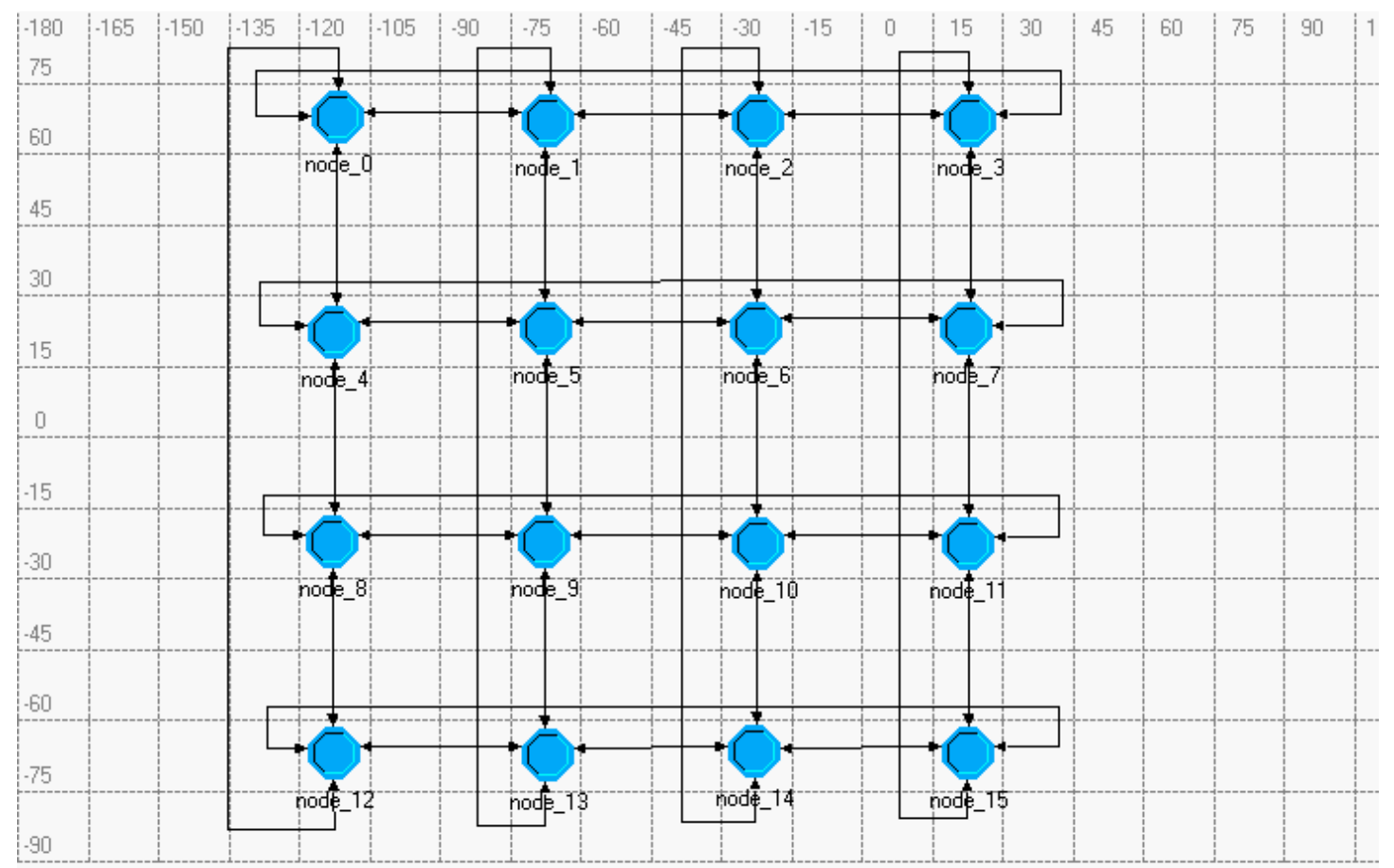

Figure 5. The $4 * 4$ Mesh Torus Network

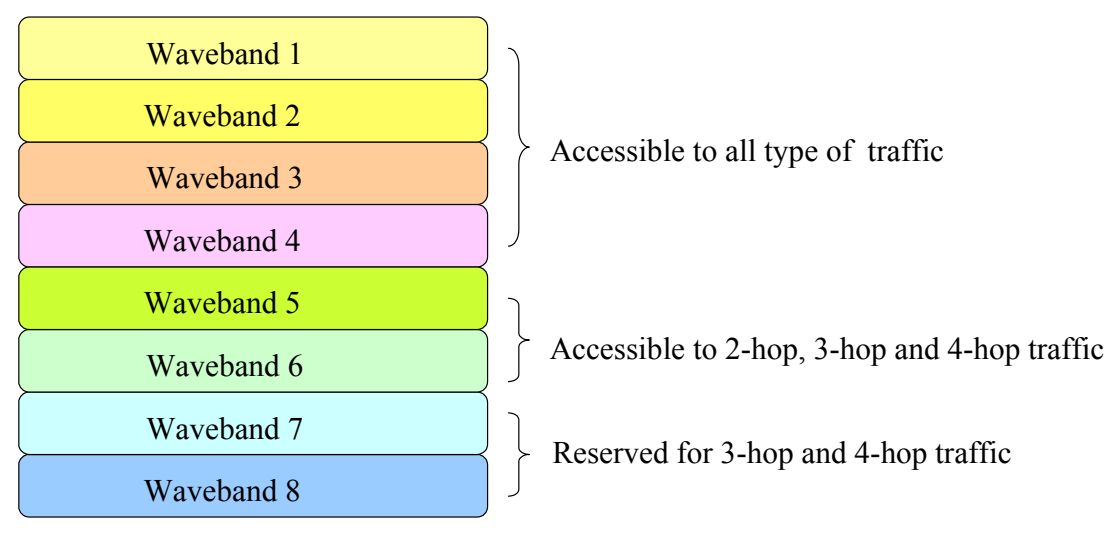

Figure 6. Waveband Access and Reservation for all type of traffic

Figures 7 and 8 show the $4 \times 4$ Mesh-Torus network's performance for all type of traffic without and with using the ClaServ method respectively. By using the ClaServ method introduced in figure 6, the blocking probability for 4-hop 
traffic is reduced by a factor of 100 , the blocking probability for 3-hop traffic is reduced by a factor of 4 , when the traffic load is 150 calls per unit time. Figure 9 shows the comparison of the average traffic blocking probability with and without using the ClaServ method in the $4 \times 4$ Mesh-Torus network. When the traffic load is 150 calls each node per unit time, the ClaServ method can reduce the average traffic blocking probability 20 times. From figures 7, 8, and 9, we can see, the ClaServ method not only can optimize the fairness problem but also can reduce the network average traffic blocking probability when the networks are required running under small blocking probability for all kind of traffic.

\section{CONCLUSION}

The Traffic Classification and Service (ClaServ) method is introduced to optimize the fairness problem and reduce the blocking probability for the network traffic. This method can be found useful as all-optical networks grow in size, seeing as more hops in the network would mean higher blocking probabilities. We have shown through simulation that the ClaServ method can reduce the blocking probability 100 times for 4-hop traffic, 4 times for 3-hop traffic, and 20 times for the overall network traffic, when the traffic load is 150 calls per unit time. The ClaServ method helps the network achieve the fairness for different traffic as well as reduce the network average traffic blocking probability when the network is required running under small traffic blocking probability.

The ClaServ method can easily been implemented into the distribute-managed signaling protocol. The traffic can be classified either at the source node or at the destination node, different service for different traffic can be provided at the destination node with the combination of the waveband access range method and the waveband reservation method.

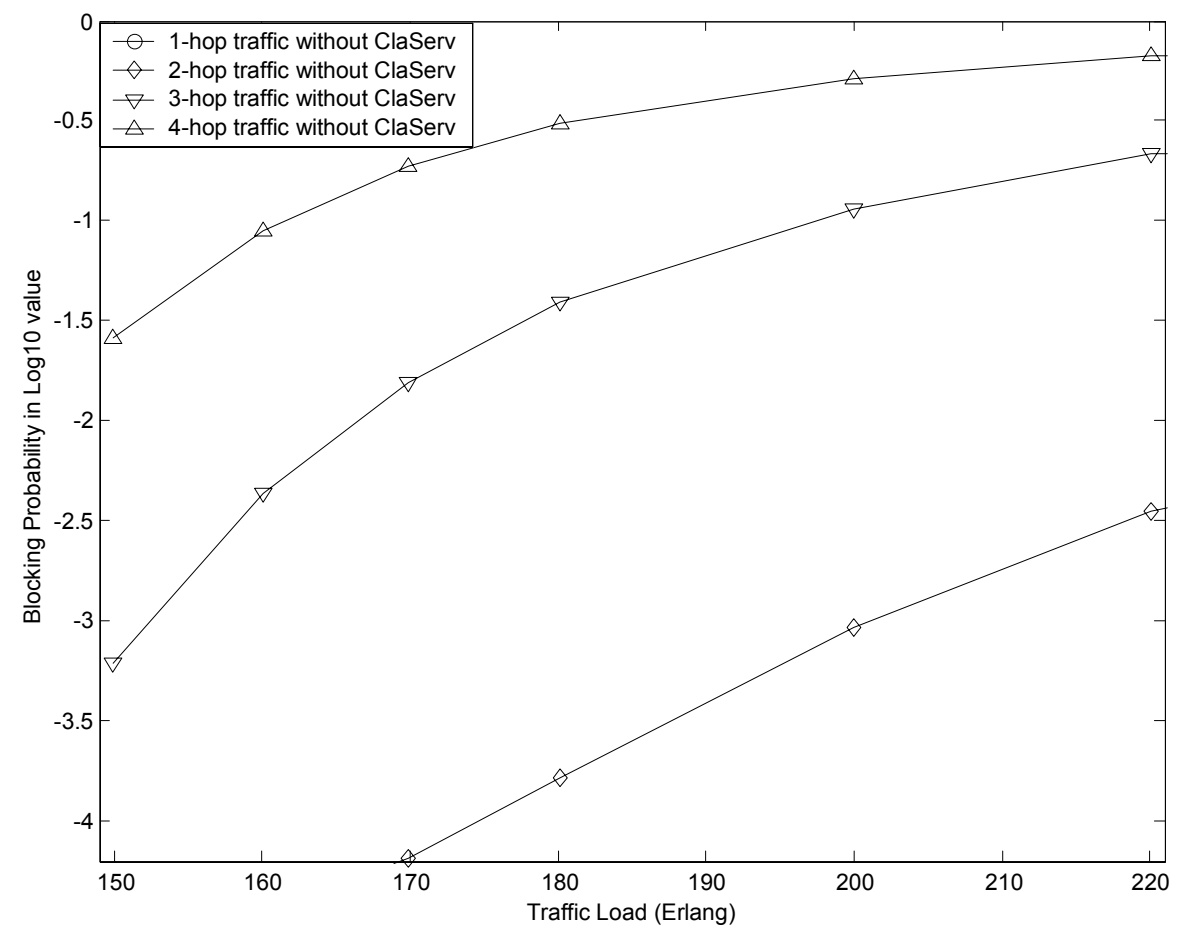

Figure 7. Performance of Different type of traffic without using ClaServ Method 


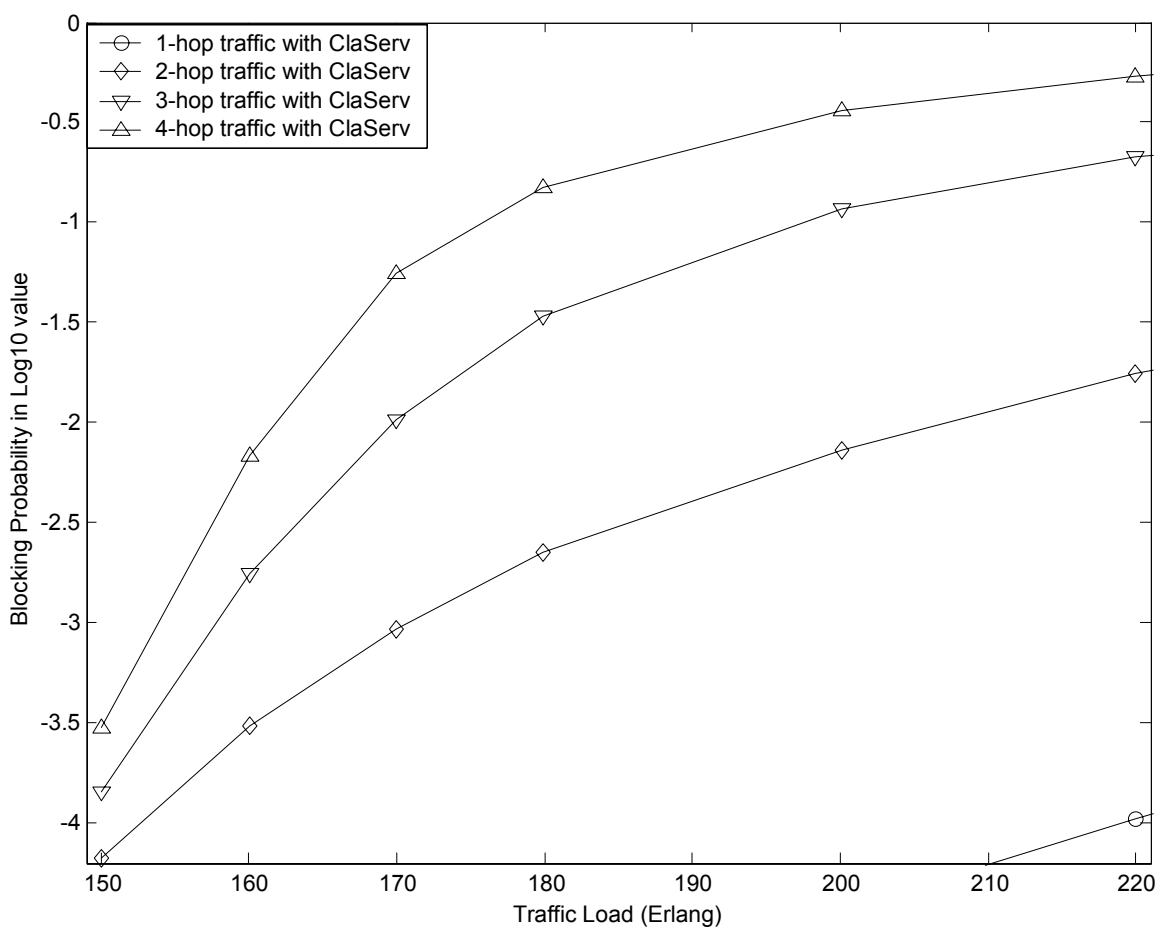

Figure 8. Performance of Different type of traffic with using ClaServ Method

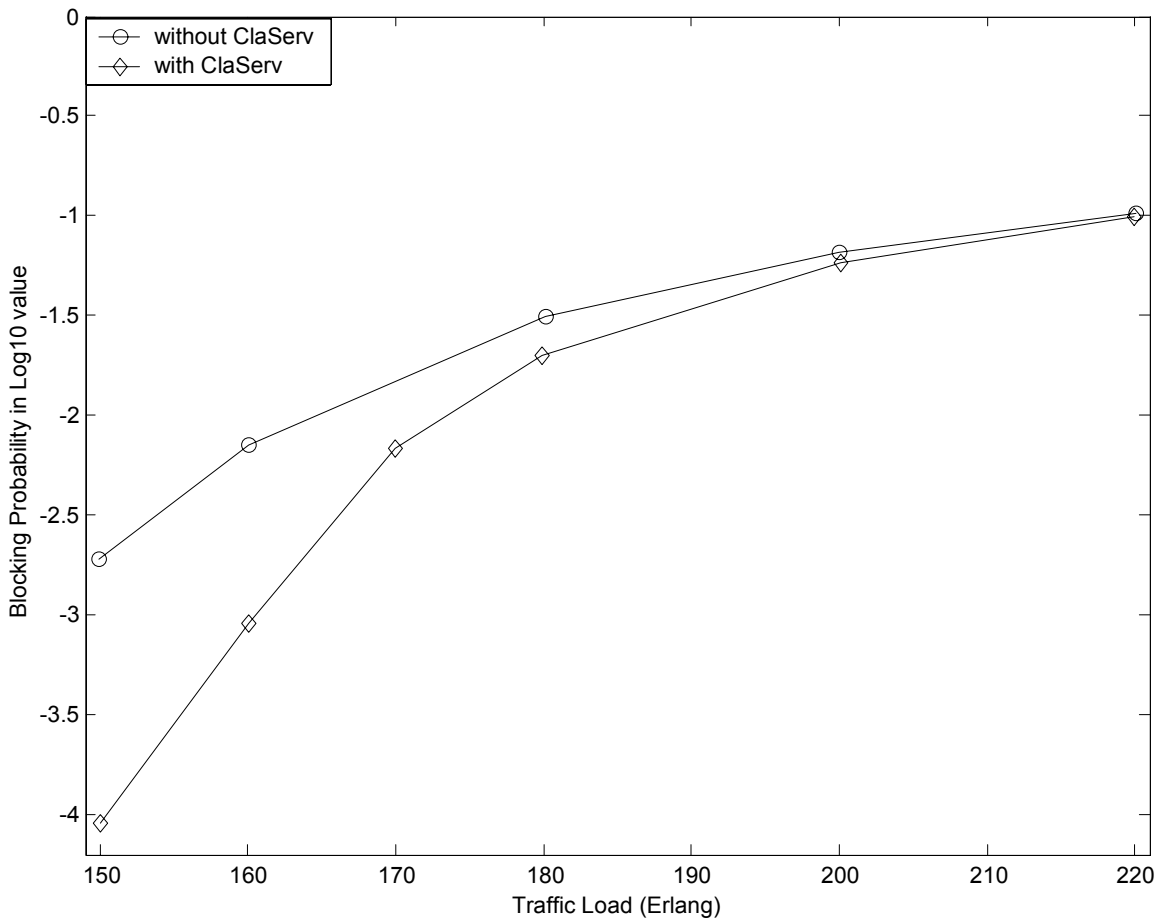

Figure 9. The Average Traffic blocking probability with and without using ClaServ Method 


\section{REFERENCES}

[1] Biswanath Mukherjee, "WDM Optical Communication Networks: Progress and Challenges", IEEE Journal on Selected Areas in communications, Vol. 18, No. 10, page (s): 1810-1824, October, 2000

[2] Paul Green, "Progress in Optical Networking", IEEE Communication Magazine, Vol. 21, No. 1, page (s): 54-64, January 2001

[3] B. Mukherjee, Optical Communication Networks. New York: McGraw-hill, 1997.

[4] Barry, R.A.; Humblet, P.A. "Models of blocking probability in all-optical networks with and without wavelength changers", Selected Areas in Communications, IEEE Journal on, Vol. 14 Issue: 5, Page(s): 858 -867, June 1996

[5] Birman, A.; Kershenbaum, A. "Routing and wavelength assignment methods in single-hop all-optical networks with blocking". INFOCOM '95. Fourteenth Annual Joint Conference of the IEEE Computer and Communications Societies. 'Bringing Information to People', Proceedings, IEEE, vol.2, Page(s): 431 -438, 1995

[6] X. Yuan, R. Melhem, R. Gupta, Y. Mei, C. Qiao, "Distributed Control Protocols For Wavelength Reservation And Their Performance Evaluation", in Photonic Networks and Communications, Vol. 1, No. 3, pp. 207-218, 1999

[7] Hui Zang, Jason P. Jue, Biswanath Mukherjee, "A Review of Routing and Wavelength Assignment Approaches For Wavelength-Routed Optical WDM Networks", Optical Networks Magazine, page (s): 47-60, January 2000 